\title{
1 Measurement of Klebsiella Intestinal Colonization Density to Assess Infection Risk
}

3 Running Title: Klebsiella colonization density and infection risk

$5 \quad$ Yuang Sun ${ }^{1}$, Alieysa Patel ${ }^{1}$, John SantaLucia $^{2}$, Emily Roberts ${ }^{3}$, Lili Zhao ${ }^{3}$, Keith S. Kaye ${ }^{4}$,

6 Krishna Rao ${ }^{4}$, and Michael A. Bachman ${ }^{1,5}$

$8 \quad{ }^{1}$ Department of Pathology, Michigan Medicine; ${ }^{2}$ DNA Software, Inc.; ${ }^{3}$ Department of

9 Biostatistics, School of Public Health; ${ }^{4}$ Division of Infectious Diseases, Department of Internal

10 Medicine, ${ }^{5}$ Department of Microbiology and Immunology, Michigan Medicine, University of

11 Michigan, Ann Arbor, MI

12

13 Corresponding Author: Michael A. Bachman

15 Contact Information:

16 mikebach@med.umich.edu

17 7510E MSRB 1

181301 Catherine

19 Ann Arbor, MI 48109 


\section{Abstract}

22 Background: Klebsiella pneumoniae and closely related species $K$. variicola and $K$.

23 quasipneumoniae are common causes of healthcare-associated infections, and patients frequently

24 become infected with their intestinal colonizing strain. To assess the association between

25 Klebsiella colonization density and subsequent infections, a case-control study was performed.

26 Methods: A multiplex qPCR assay was developed and validated to quantify Klebsiella (K.

27 pneumoniae, $K$. variicola, and K. quasipneumoniae combined) relative to total bacterial DNA

28 copies in rectal swabs. Cases of Klebsiella infection were identified based on clinical definitions

29 and having a clinical culture isolate and preceding or co-incident colonization isolate with the

30 same $w z i$ capsular sequence type. Controls were colonized patients without subsequent infection

31 and were matched 2:1 to cases based on age, sex, and rectal swab collection date. Quantitative

32 PCR (qPCR) from rectal swab samples was used to measure the association between relative

33 abundance (RA) of Klebsiella and subsequent infections.

34 Results: Klebsiella RA by qPCR highly correlated with $16 \mathrm{~S}$ sequencing $(\rho=0.79 ; P<.001)$.

35 The median Klebsiella RA in the study group was 2.6\% (interquartile range (IQR) $0.1-22.5$,

$36 \mathrm{n}=238)$, and was higher in cases $(15.7 \%$, IQR $0.93-52.6 \%, \mathrm{n}=83)$ than controls $(1.01 \%$, IQR

$37 \quad 0.02-12.8 \% ; \mathrm{n}=155 ; P<0.0001)$. After adjusting for multiple clinical covariates using inverse

38 probability of treatment weighting, subjects with a Klebsiella $\mathrm{RA}>22 \%$ had a 2.87 -fold (1.64-

$395.03, P=0.0003$ ) increased odds of infection compared to those with lower colonization density

40 levels.

41 Conclusions: Measurement of colonization density by qPCR could represent a novel approach to

42 identify hospitalized patients at risk for Klebsiella infection. 


\section{Importance}

45 Colonization by bacterial pathogens often precedes infection, and offers a window of opportunity

46 to prevent these infections. Klebsiella colonization is significantly and reproducibly associated

47 with subsequent infection, however factors that enhance or mitigate this risk in individual

48 patients are unclear. This study developed an assay to measure the density of Klebsiella

49 colonization, relative to total fecal bacteria, in rectal swabs from hospitalized patients. Applying

50 this assay to 238 colonized patients, high Klebsiella density defined as $>22 \%$ of total bacteria,

51 was significantly associated with subsequent infection. Based on widely available polymerase

52 chain reaction (PCR) technology, this type of assay could be deployed in clinical laboratories to

53 identify patients at increased risk of Klebsiella infections. As novel therapeutics are developed to

54 eliminate pathogens from the gut microbiome, a rapid Klebsiella colonization density assay

55 could identify patients who would benefit from this type of infection prevention interventions.

56

57

58 


\section{Introduction}

60 Klebsiella pneumoniae is a leading cause of healthcare-associated infections (HAIs)(1). Recent

61 studies have shown that Klebsiella variicola and Klebsiella quasipneumoniae are closely related

62 to, yet distinct species from, K. pneumoniae and cause indistinguishable infections $(2,3)$. These

63 species are part of the $K$. pneumoniae complex that together pose a serious public health threat.

64

65 Klebsiella commonly colonizes hospitalized patients and can cause bacteremia, pneumonia, and

66 urinary tract infections (UTIs). Prior studies show that Klebsiella colonization is significantly

67 associated with subsequent infections and $80 \%$ of infections in colonized patients are caused by

68 an intestinal colonizing strain $(4,5)$. Increased colonization density may increase the risk of

69 subsequent infection. For example, intestinal domination (defined as at least 30\% relative

70 colonization density) of Proteobacteria was associated with subsequent Gram-negative

71 bacteremia in patients undergoing allogeneic hematopoietic stem cell transplantation and relative

72 and absolute abundance of Enterobacterales associate interactively with infection in intensive

73 care patients $(6,7)$. In long term acute care patients, relative abundance of carbapenem-resistant

74 K. pneumoniae above $22 \%$ was a risk factor for bacteremia (8). Similarly, increased relative

75 abundance of Escherichia and Enterococcus in the gut are risk factors for corresponding

76 bacteriuria or UTI in kidney transplant patients (9). These studies indicate that in many cases

77 colonization is a necessary intermediate step before infection.

79 Understanding the association between Klebsiella colonization and subsequent infections could

80 provide opportunities for identification of high-risk patients, intervention, and ultimately

81 prevention of infection. Additionally, little is known about the association between Klebsiella gut 
82 colonization density and specific infection types, such as bacteremia, pneumonia, and UTI.

83 Measuring Klebsiella gut density and assessing gut density as a risk factor for various infections

84 may also shed light on the mechanisms of dissemination from the colonized gut to various

85 infection sites. However, the lack of a rapid and reliable assay to quantify Klebsiella relative

86 abundance in the gut has been a hindrance to both research and potential clinical implementation.

87 Here we report a qPCR-based assay that can quickly and accurately quantify Klebsiella from

rectal swab specimens. We employed this assay in a case-control study to assess Klebsiella rectal relative abundance as a risk factor for bacteremia, pneumonia or UTI in colonized patients and

90 found a significant association after adjusting for clinical variables.

\section{$\underline{\text { Results }}$}

\section{In silico Analysis}

94 To design a quantitative PCR (qPCR) assay for the K. pneumoniae complex, 31 K. pneumoniae,

K. quasipeumoniae, and $K$. variicola strains with complete genomes were selected as

Raoultella strains were selected to represent "near-neighbor exclusivity", and the human genome

100 identified as an optimal target for the assay (Table 1). An overall performance score, based on

101 primer and probe thermodynamic stabilities with their targets, as well as any off-target bindings,

102 were computed to constitute overall performance scores for each of 7 assay designs. The $y b i L$

103 assay design 1 (overall score 99.9) has a predicted probe binding score of $99.5 \%$ with all 31

104 strains in the "inclusivity" set. In regard to the primers, all 31 predicted binding scores of the 
105 forward primer (hereafter $y b i L-F$ ) are above 50.0\%, with 29 above $86.0 \%$. Twenty-four predicted

106 binding scores of the reverse primer (hereafter $y b i L-\mathrm{R}$ ) are above $95.0 \%$ while 6 are close to

$10750.0 \%$ and 1 below 50.0\%. Additionally, ybiL assay design 1 was predicted to have no

108 amplifications with any background genomes. Although its primer bindings have variations, its

109 probe binding scores are uniformly excellent. Therefore, $y b i L$ assay design 1 (hereafter $y b i L$

110 assay) was chosen for further validation. To assess the relative abundance of the $K$. pneumoniae

111 complex, the $y b i L$ assay and a previously described panbacterial qPCR assay targeting the 23S

112 rRNA gene (sepsis manuscript) were combined to construct a multiplex qPCR assay (hereafter

$113 \mathrm{Kp}$ qPCR assay). Overall, the $y b i L$ assay has good coverage of the $K$. pneumoniae complex and

114 the Kp qPCR assay provides a possible solution to quantify the $K$. pneumoniae complex in

115 clinical specimens.

117 K. pneumoniae Complex Diversity Panel

118 Eleven isolates with polymorphisms at sites of $y b i L$ primer and probe binding were picked and

119 grown overnight in LB broth. They were re-suspended in Amies media (BD ESwab ${ }^{\mathrm{TM}}$ ) and

120 normalized based on colony forming units (CFU) for DNA extraction. The lab strain ATCC

12143816 KPPR1, which contains a single mismatch identical to Kp8399, was set as reference and

122 delta-delta- $\mathrm{C}_{\mathrm{T}}\left(\mathrm{ddC}_{\mathrm{T}}\right)$ method was used to calculate the abundance of Klebsiella relative to

123 KPPR1 (set as 100\%) by qPCR. (Figure 1) Of the 11 isolates, 9 are within the range of 88

124 to $>99 \%$ relative to KPPR 1 . Although they share the same polymorphism, the abundance

125 calculation of Kp2950 was 72\% relative to KPPR1 whereas Kp6966 was 88\%. This suggests that

126 technical imprecision may be greater than systematic errors caused by polymorphisms. Taken 
127 together, the Kp qPCR assay should have accurate and consistent performance with most clinical

128 isolates despite the existence of binding variations.

\section{Specificity}

131 The Kp qPCR assay was designed to quantify K. pneumoniae, K. quasipeumoniae, and $K$.

132 variicola but not other Klebsiella species or other common bacteria in the gut microbiota. To

133 validate its specificity, K. aerogenes, K. pneumoniae subsp. ozaenae, K. oxytoca, Raoultella

134 planticola, Raoultella ornithinolytica, Escherichia coli, and Pseudomonas aeruginosa were

135 tested by the Kp qPCR assay. (Supplemental Table 1 ) K. pneumoniae KPPR1 was used as

136 positive control. Only KPPR1 and K. pneumoniae subsp. ozaenae were amplified by both $y b i L$

137 assay and 23S assay, whereas K. aerogenes, K. oxytoca, Raoultella planticola, Raoultella

138 ornithinolytica, Escherichia coli, and Pseudomonas aeruginosa strains were only amplified by

$13923 \mathrm{~S}$ assay but not $y b i L$ assay, demonstrating that $y b i L$ assay specifically amplified the designated

140 targets but not its near-neighbors or background sequences.

\section{Linearity}

143 To assess Kp qPCR assay’s linearity, KPPR1 was grown in LB broth overnight and re-suspended

144 in Amies media. A serial 10-fold dilution was made in triplicate and enumerated for CFU counts.

145 The CFU counts were close to a theoretical 10-fold dilution, as the slope was 0.9889 and $\mathrm{R}$

146 square 0.9999 . The slopes of $y b i L$ and 23S assay were both 3.37 and R squares were both 0.9994,

147 demonstrating that both assays had good linearity and efficiency. (Figure 3) 
150 To assess the precision and accuracy of Kp qPCR assay, a mixture of 89\% Bacteroides ovatus,

$151 \quad 10 \%$ KPPR 1 , and 1\% Serratia marcescens by DNA quantifications was made and diluted 10-

152 fold serially. The mixture was amplified by the Kp qPCR assay and the relative abundances of

153 Klebsiella were calculated relative to KPPR1 using the $\mathrm{ddC}_{\mathrm{T}}$ method. (Figure 4) The relative

154 abundances of Klebsiella ranged from 15.0-18.1\%, with a mean value of $17.0 \%$ (expect $10.0 \%$ ).

155 The standard deviations (hereafter SD) ranged from 0.246 to 7.386 . The quantifications by Kp

156 qPCR assay were consistent across concentrations of $4 \log _{10}$ differences. When the total DNA

157 concentrations of the mixture were over $1 \times 10^{-2} \mathrm{ng} / \mu \mathrm{l}$, the SDs of the relative abundances of

158 Klebsiella were less than $1.5 \%$. However, at $1 \times 10^{-3} \mathrm{ng} / \mu \mathrm{l}$ total DNA concentration, the assay

159 became less precise, as the SD increased to $7.39 \%$. At $1 \times 10^{-4} \mathrm{ng} / \mu 1$ total DNA concentration,

$160 y$ biL assay did not detect Klebsiella. The copy number of 23S rRNA gene is organism-specific

161 with 5, 7, and 8 copies in Bacteroides ovatus, Serratia marcescens and K. pneumoniae

162 respectively. After adjustment for these differences, the calculated relative abundance of

163 Klebsiella ranged from 10.0-12.0\%, with a mean value of $11.3 \%$ (SD 0.163 to 4.91 ),

164 demonstrating that Kp qPCR assay can accurately quantify Klebsiella from contrived samples.

165 Fortunately, a bias in the calculation based on 23S copy number in the overall population relative

166 to Klebsiella would not be expected to impact the ability to measure relative differences, as

167 demonstrated below.

\section{Accuracy: Relative differences}

170 To assess Kp qPCR assay's ability to distinguish different relative abundances of Klebsiella,

171 KPPR1 and Escherichia coli O6:K2:H1 CFT073 were mixed in Amies media according to CFU

172 counts to make $10 \%, 20 \%, 30 \%$ and $40 \%$ Klebsiella mixtures. Ten-fold serial dilutions were 
173 made from each mixture and genomic DNA of each serial dilution were isolated and then

174 amplified by Kp qPCR assay. The relative abundance of Klebsiella was calculated relative to

175 KPPR1 using the $\mathrm{ddC}_{\mathrm{T}}$ method. (Figure 5) At total DNA concentrations from $\sim 0.02-2 \mathrm{ng} / \mu \mathrm{L}$ the

176 assay was able to detect relative differences between all dilutions, and at $.002 \mathrm{ng} / \mu \mathrm{L}$ it can tell all

177 differences except between 30 and 40\% Klebsiella. At all but the lowest total bacterial

178 concentration, the assay can reliably detect $10 \%$ differences in Klebsiella relative abundance.

\section{Accuracy: In Comparison to 16S rRNA sequencing}

181 To compare the relative abundance calculated by qPCR to the gold standard of 16S rRNA

182 sequencing, 26 samples with $16 \mathrm{~S}$ rRNA sequence data were analyzed (Figure 6). Klebsiella

183 relative abundances by Kp qPCR were highly correlated with $16 \mathrm{~S}$ rRNA sequencing (Spearman's

$184 \rho=0.79 ; \mathrm{P}<.001)$. However, $16 \mathrm{~S}$ sequencing does not have the resolution to separate all species

185 and as a result, the OTU that contained $K$. pneumoniae complex also included other Klebsiella

186 spp. as well as sequences from other genera such as Enterobacter spp. This might explain why in

187 some cases Klebsiella relative abundances by Kp qPCR were significantly lower than that by

188 16S sequencing.

190 Case-control study

191 To assess the association between Klebsiella colonization density and subsequent infection, a

192 nested case-control study was performed. We previously enrolled 1978 subjects from 2087

193 separate admissions with Klebsiella colonization in a rectal swab, collected as part of routine

194 surveillance for vancomycin-resistant Enterococcus in intensive care and oncology wards

195 (Companion manuscript). Of these colonized subjects, 83 cases were identified that met clinical 
196 definitions of subsequent infection and had an infecting isolate that matched a colonizing isolate

197 on or prior to the day of infection by wzi sequence typing. This included 41 bloodstream

198 infections, 19 respiratory infections, and 23 urinary tract infections. Controls were defined as

199 colonized patients who had no documented Klebsiella infection but had a negative clinical

200 culture collected of the same type as the matching case. Each case was matched to two controls

201 based on age, sex, date of rectal swab collection and swab availability, for a total of 155 controls.

202 To find matches for every case, the criteria for age were modified for 2 cases ( \pm 20 years), and

203 the criteria for swab collection date were modified for 4 cases ( \pm 118 days). Cases had a

204 significantly higher comorbidity score, and were more likely to have exposure to diuretics,

205 vitamin D, a vasopressin blocker prior to the rectal swab. They were also more likely to have

206 been exposed to high-risk antibiotics associated with disruption of the intestinal microbiome

207 (10). Cases also had lower baseline albumin, and were more likely to have a urinary catheter or

208 feeding tube prior to the rectal swab.

210 To assess colonization density, the Kp qPCR assay was performed on all of the rectal swab

211 samples (Figure 7). The median relative abundance was 0.022 overall, with an interquartile range

212 (IQ) of 0.001-0.22 and an overall range of 0 to 1.38 . In cases, the median was 0.15 (IQ 0.009-

213 0.53) and in controls the median was 0.01 (IQ 0.0002-0.13). To determine if dominance was

214 associated with infection, while accounting for the case-control matching, a cutoff for dominance

215 was applied. The $75^{\text {th }}$ percentile of 0.22 in the overall dataset was chosen, consistent with cutoffs

216 of 0.22 and 0.3 used in previous studies $(6,8)$. Subjects with a K. pneumoniae gut colonization

217 density $>22 \%$ had a 3.34 -fold $(1.95-5.72, \mathrm{P}<0.0001)$ increased odds of infection compared to

218 those with lower colonization density levels. 
220 To adjust for patient variables associated with Klebsiella infection, inverse probability of

221 treatment weighting was used. In the overall cohort of 1978 subjects, an explanatory model for

222 invasive infection was built using baseline clinical features at the time of colonization

223 (Companion manuscript, Supplemental Table 2). The model built by purposeful selection

224 selected the following variables for inclusion: Elixhauser score, depression, diuretic use, vitamin

225 D use, use of pressors/inotropes, low serum albumin $(<2.5 \mathrm{~g} / \mathrm{dL})$, and exposure to antibiotics

226 with high risk of microbiome disruption. These variables were then used to model $K$.

227 pneumoniae gut colonization density $>22 \%$ to generate weights for the nested case-control study.

228 Using weights derived from these clinical covariates (available in 228 of 240 subjects), patients

229 with a $K$. pneumoniae gut colonization density $>22 \%$ had a 2.87 -fold $(1.64-5.03, \mathrm{P}=0.0003)$

230 increased odds of infection compared to those with lower colonization density levels. In a

231 secondary analysis by site of infection, increased relative abundance was also significantly

232 associated with bloodstream infection (OR 4.137, 95\% CI 1.448-11.818, $P=0.0084$ ), whereas

233 associations with urinary tract (OR 3.037, 95\% CI $0.571-16.17, \mathrm{P}=0.19)$ and respiratory

234 infections (OR 1.32, 95\% CI 0.38-4.565, $\mathrm{P}=0.66)$ did not reach significance.

\section{Discussion}

237 The goal of this study was to measure the association between Klebsiella colonization density

238 and subsequent infection. To develop a robust method that accurately and precisely measured the

239 relative abundance of $K$. pneumoniae, $K$. quasipneumoniae and $K$. variicola among the gut

240 microbiota, we developed a novel qPCR assay for detecting these dominant members of the $K$.

241 pneumoniae complex and combined it with measurement of 23S rRNA gene copies. Analytical 
242 validation indicated that this assay is inclusive of multiple strains of each species and is able to

243 distinguish as little as 10\% differences in relative abundance between samples. Applying this

244 assay to a case-control study of Klebsiella infections among colonized, intensive care patients

245 indicated that increased Klebsiella density is associated with subsequent infection in both

246 unadjusted and adjusted analysis.

248 The finding that Klebsiella colonization density is associated with subsequent infection raises 249 several interesting possibilities. One is that infection risk is dictated by how much Klebsiella is

250 present in the gut, independent of the varying gene content of Klebsiella strains. Indeed, we and

251 others have shown that detectable colonization is associated with infection $(4,5)$, and the lower

252 limit of detection for culture is an indirect measure of abundance in rectal swabs. However, we

253 have also demonstrated that particular Klebsiella genes are associated with infection as opposed

254 to asymptomatic colonization (11), indicating that which strain a patient is colonized with affects

255 their risk. There is likely to be an important interaction between Klebsiella gene content and

256 colonization density, where certain genes may increase gut fitness and therefore gut abundance.

257 Alternatively, there may be strains where gut abundance is increased based on microbiome

258 factors extrinsic to Klebsiella but the risk of infection is further increased by virulence genes that

259 act at the site of infection. Finally, Klebsiella strains with fitness genes that increase abundance

260 in the gut and virulence genes that act later in pathogenesis are likely to pose the greatest risk of

261 infection in colonized patients.

262

263 The main limitation of this study was the relatively small number of cases $(n=83)$. We

264 compensated for this by using a case-control design and an inverse probability of treatment 
265 weighting to account for clinical variables potentially associated with infection and intestinal

266 dominance without significant loss of statistical power (12). However, we were limited in our

267 ability to investigate associations by site of infection. Bloodstream infections were the largest

268 infection type and were independently associated with intestinal dominance. Intriguingly, the

269 point estimate for urinary tract infections (3.037) was similar to the overall odds ratio (2.87) for

270 infection. This may indicate that intestinal dominance is also associated with UTIs, perhaps

271 because a key step in pathogenesis is thought to be transit of intestinal bacteria across the

272 perineum to the urethra.

273

274 This study further supports the growing paradigm that intestinal dominance can be used to

275 predict infections in our hospitals. Previous studies have demonstrated that dominance of

276 carbapenemase-producing K. pneumoniae in long-term care patients (8), and K. pneumoniae in

277 allogeneic stem cell transplant patients (13) are associated with infection. This study evaluated a

278 more heterogeneous population of intensive care patients with a combined outcome of

279 bloodstream, respiratory or urinary tract infections and found the same association. The

280 successful use of qPCR demonstrates the feasibility of measuring relative abundance of targeted

281 pathogens in the gut using methods that are standard in clinical microbiology laboratories and

282 inexpensive relative to next-generation sequencing. This a key step in moving towards infection

283 prevention in hospitalized patients. A qPCR could be applied to detect colonization in rectal

284 swabs as well as quantify it in a single step, thereby incorporating two levels of Klebsiella

285 infection risk. Combined with the assessment of patient risk factors and perhaps targeted testing

286 for Klebsiella virulence genes, an integrated risk assessment could be performed. If this relative

287 risk is high enough, infection prevention interventions should be considered. Fortunately, safe 
and effective therapeutic strategies to eliminate gut colonization by pathogens are emerging and results from fecal transplant studies are encouraging (14). In the near future, it may be possible to assess the risk of a carbapenem-resistant Klebsiella infection at the time of hospital admission

291 and prevent it without the use of antibiotics.

\section{Methods}

295 Study design and subject enrollment. This study was approved by the Institutional Review 296 Boards of the University of Michigan Medical School (IRBMED). To assess the role of 297 Klebsiella colonization density on risk of subsequent invasive infection, we conducted a nested 298 case-control study drawn from a larger cohort of 1978 patients consecutively enrolled from 2087

299 inpatient admissions. Subjects admitted to intensive care units and oncology wards at our

300 hospital undergo routine surveillance by rectal swab culture for vancomycin-resistant

301 Enterococcus. After such testing, we collected residual media from these swabs and enrolled

302 subjects into our study if colonization with $K$. pneumoniae or K. variicola was detected by

303 selective culture on MacConkey agar and confirmed by MALDI-TOF identification (Bruker

304 MALDI Biotyper). Cases were identified from this larger cohort and matched to controls as 305 described below.

307 Case definitions. Michigan Medicine patients from intensive care units (ICU) and select wards 308 (hematology, oncology, and hematopoietic stem cell transplant) with Klebsiella colonization 309 based on a rectal swab culture and a positive Klebsiella blood, respiratory, or urine cultures were 310 identified as putative cases. Manual chart review was conducted by the study team to decide if 
311 they met clinical definitions of pneumonia or urinary tract infections (15-19). All patients with a

312 Klebsiella blood culture were considered to have an infection. For those meeting clinical case

313 definitions of infection, the clinical isolate and preceding rectal swab isolates were evaluated for

314 concordance by $w z i$ gene sequencing as previously described $(4,20)$. We have previously

315 demonstrated that wzi sequencing has similar discriminatory power to 7-gene multi-locus

316 sequence typing (4). Patients with concordant infection and colonizing isolates were considered a

317 case. Controls were defined as colonized patients who had no documented Klebsiella infection

318 but had a negative culture collected of the same type as the matching case. Cases and controls

319 were matched 1:2 based on age \pm 10 years, sex, and rectal swab collection date \pm 90 days. This

320 study was approved by the University of Michigan Institutional Review Board.

322 Samples for PCR analysis. Rectal swabs were collected using the ESwab ${ }^{\mathrm{TM}}$

323 Collection and Transport System (Becton Dickinson, Franklin Lakes, NJ, USA), which elutes the 324 sample into $1 \mathrm{~mL}$ Amies medium. Unless specified otherwise, contrived samples that were used

325 in PCR analysis were eluted in the Amies medium as well. The $89 \%$ Bacteroides ovatus, $10 \%$

326 KPPR1, and 1\% Serratia marcescens mixtures were suspended in $\mathrm{ddH}_{2} \mathrm{O}$.

328 Bacterial DNA extraction. Genomic DNA was isolated using the MagAttract

329 PowerMicrobiome DNA/RNA kit (Qiagen, Germantown, MD, USA) on the epMotion ${ }^{\circledR} 5075$

330 liquid handler (Eppendorf, Hauppauge, NY, USA). A volume of $100 \mathrm{uL}$ was added into the bead

331 plate for each rectal swab and contrived sample. Subsequent steps of DNA extraction were

332 conducted following the manufacturer's instructions. Bacteroides ovatus, KPPR1, and Serratia 
marcescens cultures were extracted using DNeasy Blood \& Tissue Kits (Qiagen, Germantown, MD, USA) following the manufacturer's instruction for Gram-negative bacteria.

In silico assay design. PanelPlex ${ }^{\mathrm{TM}}$ (DNA Software, Ann Arbor, MI, USA) in silico analysis

338 selected as "inclusivity", 8 K. oxytoca and Raoultella strains as "near-neighbor exclusivity", and

339 the human genome and common members of the gut microbiome as background sequences that

340 should not be detected by the assay (Supplemental Dataset 1). PanelPlex ${ }^{\mathrm{TM}}$ utilizes

341 ThermoBLAST ${ }^{\mathrm{TM}}$ technology to scan for thermodynamically stable off-target hybridizations that 342 cause false-positive tests.

344 Quantitative PCR assay. Real-time PCR was performed using primers (Integrated DNA

345 Technologies, Coralville, IA, USA) and probes (Thermo Fisher Scientific, Waltham, MA, USA)

346 with sequences and concentrations listed in Table 1 in combination with QuantiTect Multiplex

347 PCR Kit (Qiagen, Germantown, MD, USA). PMAxx (Biotium, Fremont, CA, USA) with a final

348 concentration of $6 \mathrm{uM}$ was added. A volume of $5 \mu \mathrm{L}$ was used for each template. The final

349 reaction volume was $25 \mu \mathrm{L}$. Prior to template addition, the reaction mixture was incubated for 10

350 minutes at room temperature and then treated in a Biotium PMA-Lite LED photolysis device for

35110 minutes. PCR conditions were $50^{\circ} \mathrm{C}$ for 2 minutes, $95^{\circ} \mathrm{C}$ for 15 minutes, then 40 cycles of

$35294^{\circ} \mathrm{C}$ for 1 minute and $60^{\circ} \mathrm{C}$ for 1 minute on a QuantStudio 3 real-time thermocycler (Thermo

353 Fisher Scientific, Waltham, MA, USA). KPPR1 genomic DNA was used as positive control and

$354 \quad 100 \%$ reference for calculating Klebsiella relative abundances. Relative abundance was 
355 calculated using the $\mathrm{ddC}_{\mathrm{T}}$ method, i.e. Relative abundance $\left.=2^{\left(d \mathrm{C}_{\mathrm{T}} \text { sample }\right.}-d \mathrm{C}_{\mathrm{TKPPR} 1}\right) \times 100 \%$,

356 where $d \mathrm{C}_{\mathrm{T}}=\mathrm{C}_{\mathrm{T}_{23 S}}-\mathrm{C}_{\mathrm{T}}{ }_{y b i L}$.

358 Statistical Analysis. Linearity was validated by linear regression. Spearman's rank correlation

359 coefficient was used for correlation between Kp qPCR and 16S rRNA sequencing. One-way

360 ANOVA and Tukey's post test was performed to compare each dilution of the KPPR1 and

361 Escherichia coli O6:K2:H1 CFT073 mixtures. Statistical analysis was performed by Prism 8

362 (GraphPad, San Diego, CA, USA).

364 Clinical modeling.

365 Conditional logistic regression was used to study the effect of relative abundance of colonization

366 on Klebsiella infection, while accounting for the case-control matching. Unadjusted analysis was

367 performed after dichotomizing the relative abundance at the third quartile of $22 \%$. To adjust for

368 patient variables associated with Klebsiella infection, an inverse probability of treatment

369 weighting approach was used. However, given the smaller sample size in our nested case-control

370 study, we turned to the larger cohort from which our subjects were derived to identify most

371 robustly the clinical variables that best explain risk of infection (Companion manuscript). First,

372 using the increased power afforded by the overall cohort of 1978 subjects, an explanatory

373 unconditional logistic regression model for invasive infection was built using baseline clinical

374 features at the time of colonization. The model was built by purposeful selection, a common

375 technique(21). Briefly, purposeful selection begins with an unadjusted analysis of each variable

376 to select candidates with statistically significant associations with the outcome, and these are

377 included in the starting set of covariates for the multivariable model. Iteratively, covariates are 
378 then removed from the model if they are non-significant $(P>.05)$ and not a confounder (i.e. do

379 not affect the estimate of other variables' coefficients by at least $20 \%$ ). A change in a parameter

380 estimate above the specified level indicates that the excluded variable was important in the sense

381 of providing a needed adjustment for one or more of the variables remaining in the model (i.e. it

382 should be retained even if not significant). The resulting model contains significant covariates

383 and other confounders, and then variables not included are added back one at a time. Once again

384 the model is iteratively reduced as before but only for the variables that were additionally added.

385 At the end of this final step, we are left with a multivariable model for Klebsiella infection drawn

386 from the larger cohort of subjects with rectal Klebsiella colonization. The variables selected for

387 inclusion by this method were then used to generate propensity scores for Klebsiella colonization

388 density $>22 \%$, but only for subjects in the nested case-control study, again via unconditional

389 logistic regression. The propensity scores were then used to generate weights for the IPTW

390 process and subsequent weighted conditional logistic regression for Klebsiella infection

391 The inverse of these propensity scores were then used as weights in the subsequent weighted

392 conditional logistic regression for Klebsiella infection with robust standard errors. Both

393 unadjusted and adjusted analysis were conducted using proc survveylogistic procedure in SAS

394 (version 9.4), and covariate balance was assessed using the cobalt package in R.

396 Data Availability:

397 16S sequencing samples from rectal swabs PR08714, PR11216, PR05497, PR09929, PR10907,

398 PR05713, PR06107, PR08411, PR08133, PR05629, PR08147, PR07331, PR12066, PR07876,

399 PR08427, PR07976, PR08661, PR05017, PR08962, PR09113, PR08102, PR09612, PR08748,

400 PR08048, PR06316, PR10214 are available in the sequence read archive (PRJNA641414). 
bioRxiv preprint doi: https://doi.org/10.1101/2021.02.16.431551; this version posted February 17, 2021. The copyright holder for this preprint (which was not certified by peer review) is the author/funder, who has granted bioRxiv a license to display the preprint in perpetuity. It is made available under aCC-BY-NC-ND 4.0 International license.

401 Deidentified data from human subjects may be made available upon request, pending approval

402 from the University of Michigan Institutional Review Board.

403

404 


\section{Acknowledgements}

406 MAB would like to thank Caitlyn Holmes for assistance with figure formatting.

408 Research reported in this publication was supported by the National Institute Of Allergy And

409 Infectious Diseases of the National Institutes of Health under Award Number R01AI125307. The

410 content is solely the responsibility of the authors and does not necessarily represent the official

411 views of the National Institutes of Health. 
412 Table 1. Primers and probes used in the study

\begin{tabular}{|l|l|l|}
\hline Oligonucleotide & Final & Sequence (5' to 3') \\
& Ronc. & \\
\hline ybiL_Probe & $200 \mathrm{nM}$ & FAM-CGTCCACAGCGTAAAGGCATGTT-MGB \\
\hline 23S_Probe & $200 \mathrm{nM}$ & VIC-CCTAAGGTAGCGAAATTCCTTGT-MGB \\
\hline ybiL-F & $400 \mathrm{nM}$ & AACGTAGCGCAGGATGGATCTTCCG \\
\hline ybiL-R & $400 \mathrm{nM}$ & GACAGATCGCTGGTGGCCTGATA \\
\hline $23 S-F$ & $400 \mathrm{nM}$ & ATTACGCCATTCGTGCAGGTCGGA \\
\hline $23 S-R$ & $400 \mathrm{nM}$ & TAAACGGCGGCCGTAACTATAACGGT \\
\hline
\end{tabular}

413

414

415

416

417

418 
Table 2. Patient Demographics

\begin{tabular}{|c|c|c|c|}
\hline & No. $(\%)$ or $\mathrm{me}$ & $+/-\mathrm{SD}$ & \\
\hline Variable & Cases $(n=83)$ & Controls $(\mathrm{n}=155)$ & $\begin{array}{l}\text { P-value } \\
\text { (logistic } \\
\text { regression) }\end{array}$ \\
\hline Age & $60.08 \pm 12.90$ & $59.43 \pm 12.29$ & 0.759 \\
\hline gender (Male) & $44(53.0)$ & $83(53.5)$ & $>.99$ \\
\hline Race (White) & $70(84.3)$ & $122(78.7)$ & 0.368 \\
\hline Elixhauser Comorbidity Score & $7.53 \pm 3.25$ & $6.62 \pm 3.12$ & 0.05 \\
\hline Weighted Elixhauser Score & $22.40 \pm 11.53$ & $19.36 \pm 11.89$ & 0.104 \\
\hline Depression & $29(34.9)$ & $40(25.8)$ & 0.203 \\
\hline Diuretic & $30(36.1)$ & $36(23.2)$ & 0.03 \\
\hline Vitamin D & $18(21.7)$ & $18(11.6)$ & 0.032 \\
\hline Vasopressin blocker & $19(22.9)$ & $15(9.7)$ & 0.008 \\
\hline Broad-spectrum antibiotic $^{1}$ & $30(36.1)$ & $30(19.4)$ & 0.005 \\
\hline Baseline albumin (g/dL) & $2.53 \pm 0.71$ & $2.78 \pm 0.73$ & 0.008 \\
\hline Albumin $>=2.5(\mathrm{~g} / \mathrm{dL})$ & $46(55.4)$ & $112(72.3)$ & 0.003 \\
\hline urinary catheter & $60(72.3)$ & $86(55.5)$ & 0.016 \\
\hline feeding tube & $7(8.43)$ & $3(1.94)$ & 0.023 \\
\hline ventilator & $35(42.2)$ & $66(42.6)$ & $>.99$ \\
\hline central line & $34(41.0)$ & $74(47.7)$ & 0.301 \\
\hline \multicolumn{4}{|c|}{$\begin{array}{l}{ }^{1} \text { Defined as exposure to any of the following in the } 90 \text { days prior to Klebsiella } \\
\text { colonization: third- or fourth-generation cephalosporins, fluoroquinolones, } \\
\text { lincosamides, } \beta \text {-lactam / } \beta \text {-lactamase inhibitor combinations, oral vancomycin, and } \\
\text { carbapenems (10). }\end{array}$} \\
\hline
\end{tabular}




\section{References}

423

424 1. Magill SS, Edwards JR, Bamberg W, Beldavs ZG, Dumyati G, Kainer MA, Lynfield R,

425 Maloney M, McAllister-Hollod L, Nadle J, Ray SM, Thompson DL, Wilson LE, Fridkin infections. N Engl J Med 370:1198-208.

2. Holt KE, Wertheim H, Zadoks RN, Baker S, Whitehouse CA, Dance D, Jenney A,

436 3. Long SW, Linson SE, Ojeda Saavedra M, Cantu C, Davis JJ, Brettin T, Olsen RJ. 2017. Whole-Genome Sequencing of Human Clinical Klebsiella pneumoniae Isolates Reveals Misidentification and Misunderstandings of Klebsiella pneumoniae, Klebsiella variicola, and Klebsiella quasipneumoniae. mSphere 2:e00290-17.

4. Martin RM, Cao J, Brisse S, Passet V, Wu W, Zhao L, Malani PN, Rao K, Bachman MA. 2016. Molecular Epidemiology of Colonizing and Infecting Isolates of Klebsiella pneumoniae. mSphere 1:e00261-16.

443 5. Gorrie CL, Mirceta M, Wick RR, Edwards DJ, Thomson NR, Strugnell RA, Pratt NF, 
447 6. Taur Y, Xavier JB, Lipuma L, Ubeda C, Goldberg J, Gobourne A, Lee YJ, Dubin KA, hematopoietic stem cell transplantation. Clin Infect Dis 55:905-14.

451 7. Rao K, Seekatz A, Bassis C, Sun Y, Mantlo E, Bachman MA. 2020. Enterobacterales Infection after Intestinal Dominance in Hospitalized Patients. mSphere 5:pii: 5/4/e0045020.

8. Shimasaki T, Seekatz A, Bassis C, Rhee Y, Yelin RD, Fogg L, Dangana T, Cisneros EC, term Acute Care Hospital Patients. Clin Infect Dis 68:2053-2059.

9. Magruder M, Sholi AN, Gong C, Zhang L, Edusei E, Huang J, Albakry S, Satlin MJ, Commun 10:5521. 
11. Martin RM, Cao J, Wu W, Zhao L, Manthei DM, Pirani A, Snitkin E, Malani PN, Rao K, Bachman MA. 2018. Identification of Pathogenicity-Associated Loci in Klebsiella pneumoniae from Hospitalized Patients. mSystems 3:e00015-18.

471 12. Haukoos JS, Lewis RJ. 2015. The Propensity Score. JAMA 314:1637-8.

472 13. Stoma I, Littmann ER, Peled JU, Giralt S, van den Brink MRM, Pamer EG, Taur Y. 2020. Compositional flux within the intestinal microbiota and risk for bloodstream infection with gram-negative bacteria. Clin Infect Dis doi:10.1093/cid/ciaa068.

475 14. Gargiullo L, Del Chierico F, D'Argenio P, Putignani L. 2019. Gut Microbiota Modulation for Multidrug-Resistant Organism Decolonization: Present and Future Perspectives. Front Microbiol 10:1704.

15. American Thoracic S, Infectious Diseases Society of A. 2005. Guidelines for the management of adults with hospital-acquired, ventilator-associated, and healthcareassociated pneumonia. Am J Respir Crit Care Med 171:388-416.

481 16. CDC. 2016. Urinary tract infection (catheter-associated urinary tract infection [CAUTI] and non-catheter-associated urinary tract infection [UTI]) and other urinary system

484 17. Hooton TM, Bradley SF, Cardenas DD, Colgan R, Geerlings SE, Rice JC, Saint S, Schaeffer AJ, Tambayh PA, Tenke P, Nicolle LE, Infectious Diseases Society of A. 2010. Diagnosis, prevention, and treatment of catheter-associated urinary tract infection in adults: 2009 International Clinical Practice Guidelines from the Infectious Diseases

489 18. Mandell LA, Wunderink RG, Anzueto A, Bartlett JG, Campbell GD, Dean NC, Dowell 
Diseases Society of A, American Thoracic S. 2007. Infectious Diseases Society of America/American Thoracic Society consensus guidelines on the management of community-acquired pneumonia in adults. Clin Infect Dis 44 Suppl 2:S27-72.

494 19. CDC. 2014. National Healthcare Safety Network device-associated module: ventilatorassociated events.Centers for Disease Control and Prevention. Centers for Disease Control and Prevention, Atlanta, GA.

497 20. Brisse S, Passet V, Haugaard AB, Babosan A, Kassis-Chikhani N, Struve C, Decre D. 2013. wzi Gene sequencing, a rapid method for determination of capsular type for Klebsiella strains. J Clin Microbiol 51:4073-8.

500 21. Bursac Z, Gauss CH, Williams DK, Hosmer DW. 2008. Purposeful selection of variables in logistic regression. Source Code Biol Med 3:17. 


\section{Figure Legends.}

505 Figure 1. The Kp qPCR assay accurately quantifies $K$. pneumoniae, K. variicola and $K$.

506 quasipneumoniae. Eleven Klebsiella clinical isolates were extracted and amplified by Kp qPCR

507 assay, each with 3 technical replicates. A. Quantification of each isolate relative to KPPR1 set as

$508100 \%$. B. The alignment of the amplicons of the 11 isolates with Kp qPCR assay primers and

509 probe.

511 Figure 2: The Kp qPCR assay has the accuracy, precision and linearity to distinguish small

512 differences in Klebsiella relative abundance. Linearity was assessed with serial dilutions of

513 KPPR1 genomic DNA ( $\mathrm{n}=3$ technical replicates). Linear regression of $\mathrm{Ct}$ values from the $y b i L$

514 assay (blue) and 23S (red) is shown (A). Precision and accuracy was assessed with serial

515 dilutions of a mixture of 89\% Bacteroides ovatus, 10\% K. pneumoniae KPPR1, and 1\% Serratia

516 marcescens genomic DNA (3 technical replicates). Mean and SD of both direct and adjusted

517 quantifications, after consideration of $23 \mathrm{~S}$ gene copy number variations, are shown B). The

518 ability to discern relative differences is shown using serial dilutions of mixtures of KPPR 1 and

519 Escherichia coli CFT073 (3 technical replicates). For each dilution, one-way ANOVA was

520 performed $(P<0.0001$ for all $)$ and Tukey's post test was performed (* for each comparison out of

521 six with $P<0.05)(\mathrm{C})$. Accuracy was compared to $16 \mathrm{~S}$ rRNA sequencing using OTU 4 that

522 contains Klebsiella. The correlation between Klebsiella relative abundance by Kp qPCR and

523 OTU4 of 16S rRNA sequencing analysis that contains Klebsiella was measured by Spearman's

524 rank correlation coefficient on 26 rectal swab samples (D). 
526 Figure 3. Increased relative abundance of Klebsiella is associated with subsequent infection. The

527 relative abundance of Klebsiella in rectal swabs as measured by the Kp qPCR assay is shown for

528238 specimens, with 83 cases matched 1:2 to 155 controls based on age, sex and date of swab

529 collection. Median and interquartile ranges are shown. **** $\mathrm{P}<0.0001$ by unadjusted conditional

530 logistic regression. 
A

Diversity panel

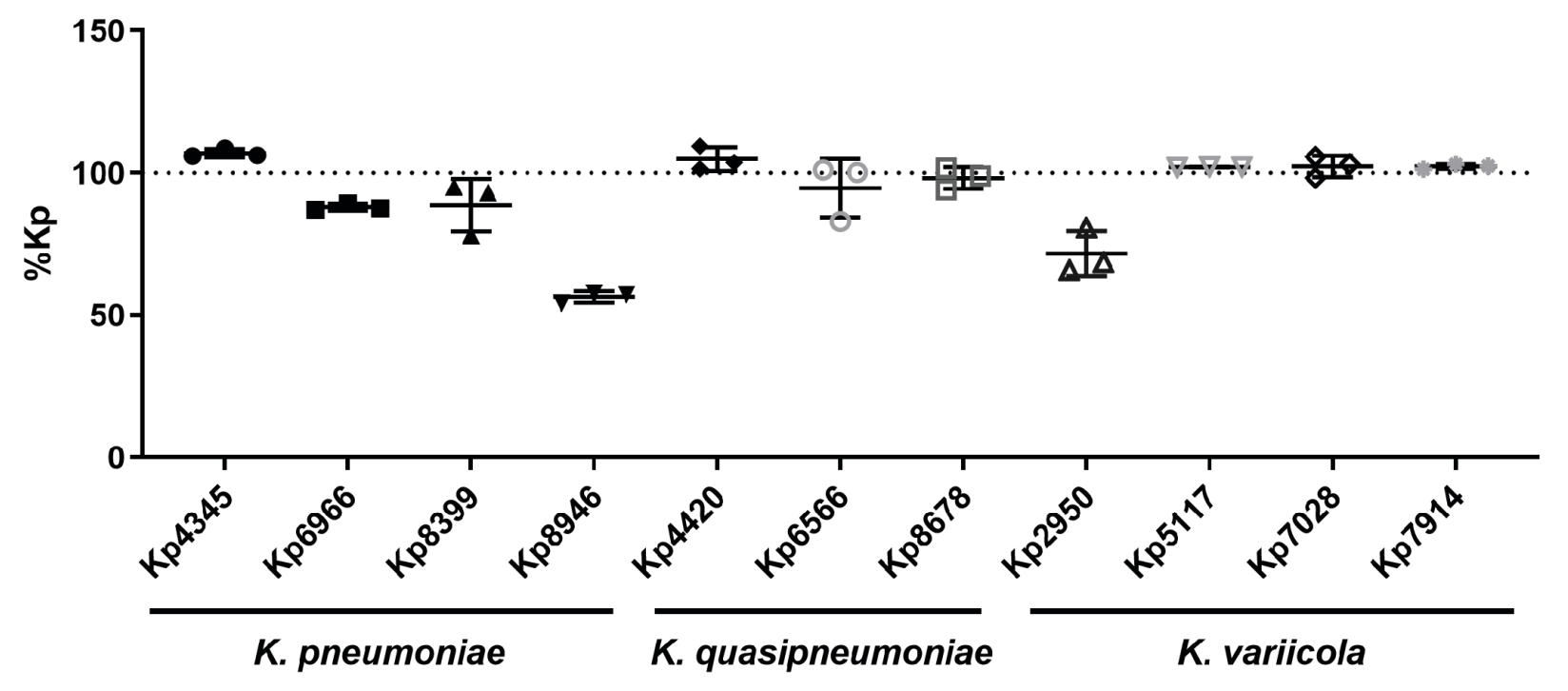

B

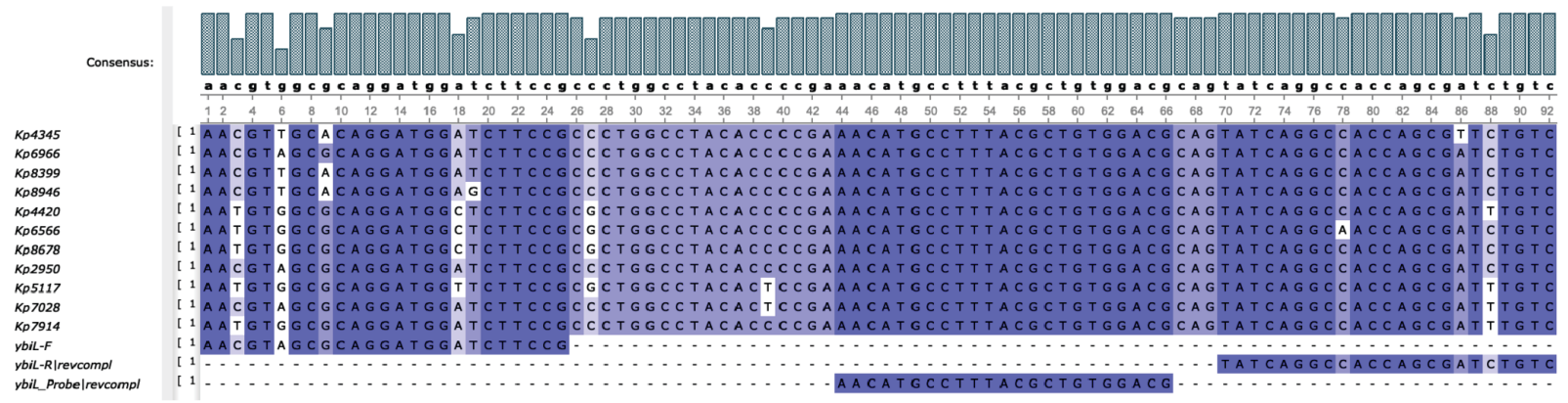

534 Figure 1. The Kp qPCR assay accurately quantifies $K$. pneumoniae, $K$. variicola and $K$.

535 quasipneumoniae. Eleven Klebsiella clinical isolates were extracted and amplified by Kp qPCR

536 assay, each with 3 technical replicates. A. Quantification of each isolate relative to KPPR1 set as

$537100 \%$. B. The alignment of the amplicons of the 11 isolates with Kp qPCR assay primers and

538 probe. 


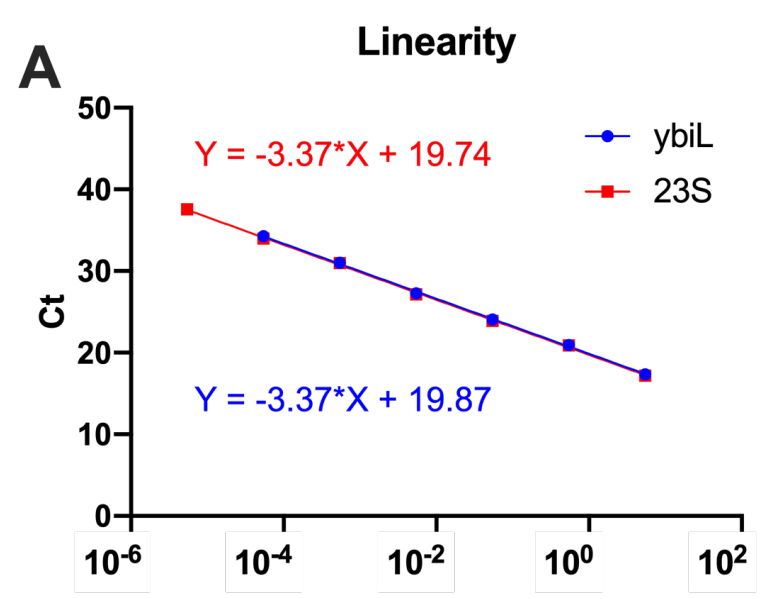

Total DNA ng/ul

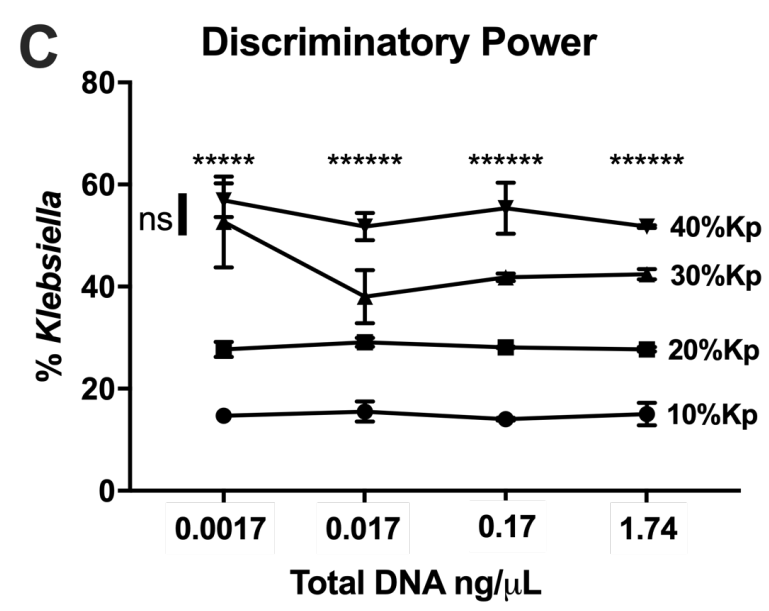

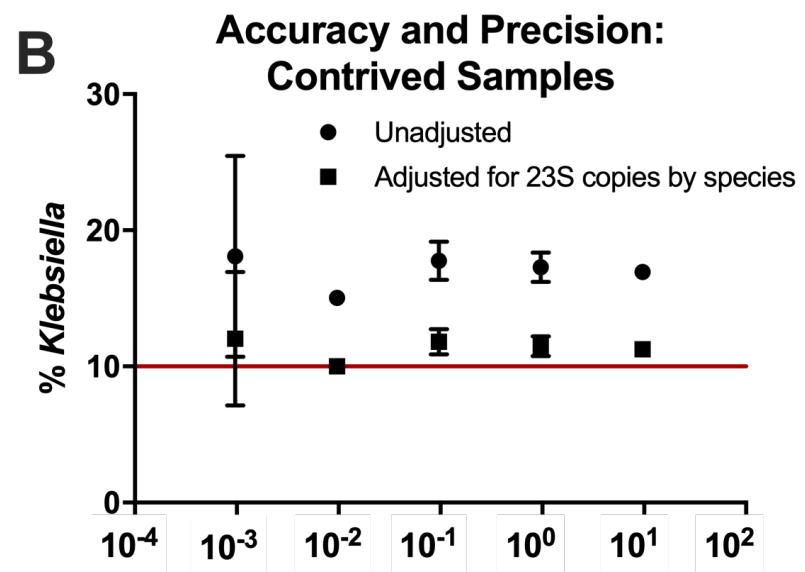

Total DNA ng/ul

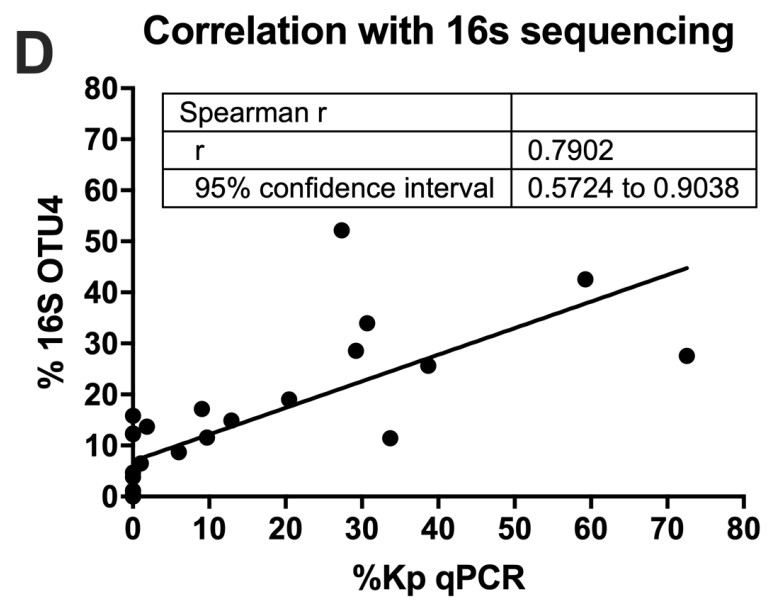

543 Figure 2: The Kp qPCR assay has the accuracy, precision and linearity to distinguish small

544 differences in Klebsiella relative abundance. Linearity was assessed with serial dilutions of

545 KPPR1 genomic DNA ( $\mathrm{n}=3$ technical replicates). Linear regression of $\mathrm{Ct}$ values from the $y b i L$

546 assay (blue) and 23S (red) is shown (A). Precision and accuracy were assessed with serial

547 dilutions of a mixture of 89\% Bacteroides ovatus, 10\% K. pneumoniae KPPR1, and 1\% Serratia

548 marcescens genomic DNA (3 technical replicates). Mean and SD of both direct and adjusted

549 quantifications, after consideration of 23S gene copy number variations, are shown B). The

550 ability to discern relative differences is shown using serial dilutions of mixtures of KPPR1 and 
551 Escherichia coli CFT073 (3 technical replicates). For each dilution, one-way ANOVA was

552 performed $(P<0.0001$ for all) and Tukey's post test was performed (* for each comparison out of

553 six with $P<0.05)(\mathrm{C})$. Accuracy was compared to $16 \mathrm{~S}$ rRNA sequencing using OTU 4 that

554 contains Klebsiella. The correlation between Klebsiella relative abundance by Kp qPCR and

555 OTU4 of 16S rRNA sequencing analysis that contains Klebsiella was measured by Spearman's

556 rank correlation coefficient on 26 rectal swab samples (D). 


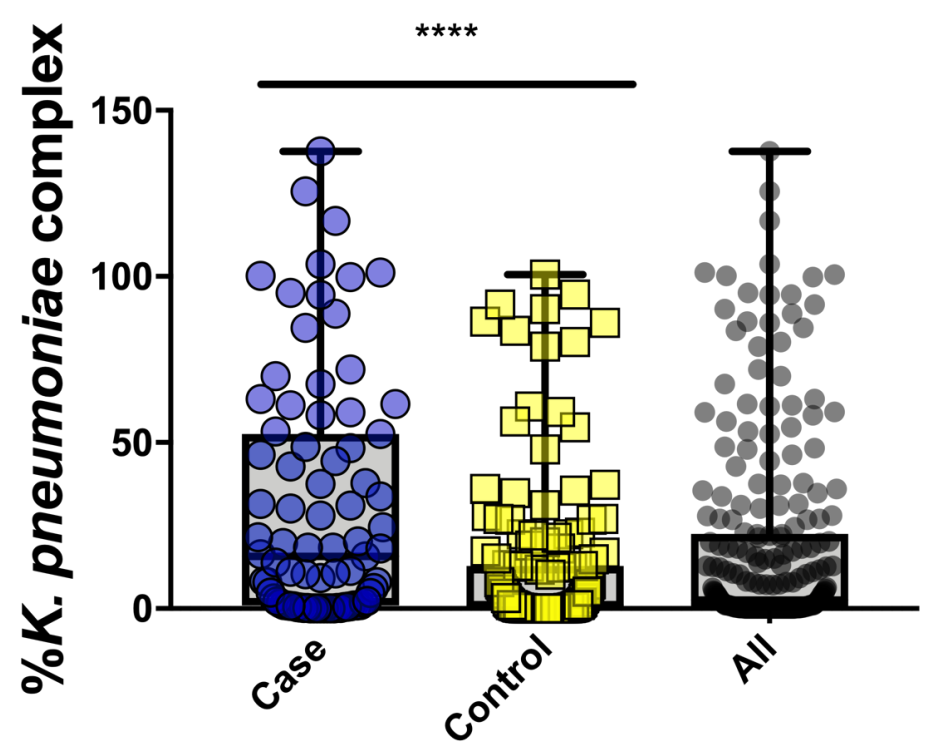

\begin{tabular}{|l|l|l|l|}
\hline & Case & Control & All \\
\hline Number of values & 83 & 155 & 238 \\
\hline & & & \\
\hline Minimum & 0 & 0 & 0 \\
\hline $25 \%$ Percentile & 0.932 & 0.017 & 0.101 \\
\hline Median & 15.74 & 1.009 & 2.605 \\
\hline $75 \%$ Percentile & 52.61 & 12.8 & 22.54 \\
\hline Maximum & 137.7 & 100.6 & 137.7 \\
\hline & & & \\
\hline Mean & 30.45 & 11.9 & 18.37 \\
\hline Std. Deviation & 36.09 & 22.79 & 29.44 \\
\hline Std. Error of Mean & 3.961 & 1.831 & 1.908 \\
\hline
\end{tabular}

560 Figure 3. Increased relative abundance of Klebsiella is associated with subsequent infection. The

561 relative abundance of Klebsiella in rectal swabs as measured by the Kp qPCR assay is shown for

562238 specimens, with 83 cases matched 1:2 to 155 controls based on age, sex and date of swab

563 collection. Median and interquartile ranges are shown. **** $\mathrm{P}<0.0001$ by unadjusted conditional

564 logistic regression. 